\title{
CKM and CP Constraints from B-Decays
}

\author{
Shohei Nishida* \\ High Energy Accelerator Research Organization (KEK) \\ E-mail: Shohei.nishidadkek.jp
}

Study of $C P$ violation and the precise measurements of the Cabibbo-Kobayashi-Maskawa (CKM) matrix provide test of the Standard Model. Recent measurements on angles and sides of the unitarity triangle such as $\phi_{1}, \phi_{3}, \phi_{s},\left|V_{c b}\right|$ from ATLAS, BaBar, Belle and LHCb experiments are summarized. LHCb has performed amplitude analyses on 3 body $B$ decays and has studied the source of local $C P$ asymmetry. This study is also mentioned in this proceedings.

XXIX International Symposium on Lepton Photon Interactions at High Energies - LeptonPhoton2019 August 5-10, 2019

Toronto, Canada

${ }^{*}$ Speaker. 


\section{Introduction}

$C P$ violation in the Standard Model (SM) occurs though the complex phase in the quark mixing matrix. This matrix, called Cabibbo-Kobayashi-Maskawa (CKM) matrix, is a unitary matrix and can be expressed as

$$
V_{C K M}=\left(\begin{array}{lll}
V_{u d} & V_{u s} & V_{u b} \\
V_{c d} & V_{c s} & V_{c b} \\
V_{t d} & V_{t s} & V_{t b}
\end{array}\right)=\left(\begin{array}{ccc}
1-\lambda^{2} / 2 & \lambda & A \lambda^{3}(\rho-i \eta) \\
-\lambda & 1-\lambda^{2} / 2 & A \lambda^{2} \\
A \lambda^{3}(1-\rho-i \eta) & -A \lambda^{2} & 1
\end{array}\right) .
$$

From the unitarity of the matrix, one can derive a relation

$$
V_{u d} V_{u b}^{*}+V_{c d} V_{c b}^{*}+V_{t d} V_{t b}^{*}=0
$$

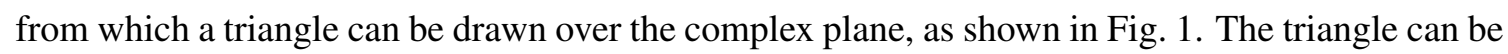
overconstrained by measurements of the three angles $\phi_{1}=\beta, \phi_{2}=\alpha, \phi_{3}=\gamma$ and the sides, giving a test of the SM. The present constraint is shown in Fig. $\square$. So far the measurements are generally consistent; however, there is still room that some New Physics (NP) effect could exist.

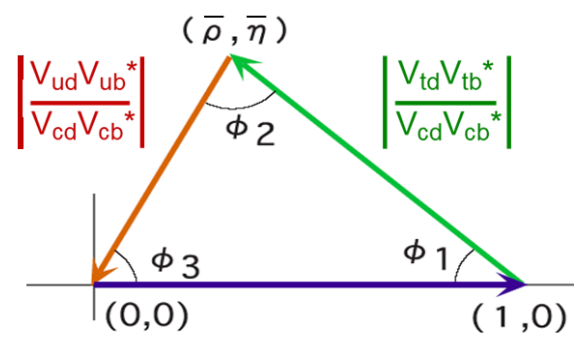

Figure 1: Definition of the Unitarity Triangle.

Many of the measurements to constrain CKM matrix have been done with flavor physics experiments. Belle and Babar are the $B$ factory experiments with an asymmetric $e^{+} e^{-}$collider. They already completed the operation, but still produce new results from their data already taken. $\mathrm{LHCb}$ is a $b$-physics experiment at LHC, and is now leading the study with $B$ and $B_{s}$ decays. $B$ physics is also studied at ATLAS and CMS experiment at LHC. Belle II experiment, the successor of Belle aiming at 50 times higher integrated luminosity, has started its physics run in 2019, and it will provides new results in near future.

In this proceedings, recent measurements on $\phi_{1}, \phi_{3}$, a weak phase $\phi_{s}$ (discussed in Sec. [1), $\left|V_{c b}\right|$ as well as amplitude analyses of 3 body $B$ decays from LHCb are summarized.

\section{Measurement of $\phi_{1}(=\beta)$}

The measurement of $\sin \left(2 \phi_{1}\right)$ has been one of the most important topic in $B$ factories, and it has been precisely measured as $\sin \left(2 \phi_{1}\right)=0.699 \pm 0.017$ [四] with $b \rightarrow c \bar{c} s$ tree processes. Measurements of the same value with other processes with penguin loops such as $b \rightarrow q \bar{q} s(q=u, d, s)$ are useful to probe non-SM contribution in the penguin loop. Recently, Belle has performed measurents of the $C P$ violation parameter $S$ in $B^{0} \rightarrow J / \psi \pi^{0}$ and $B^{0} \rightarrow \pi^{0} \pi^{0} K_{S}^{0}$. The former proceeds 


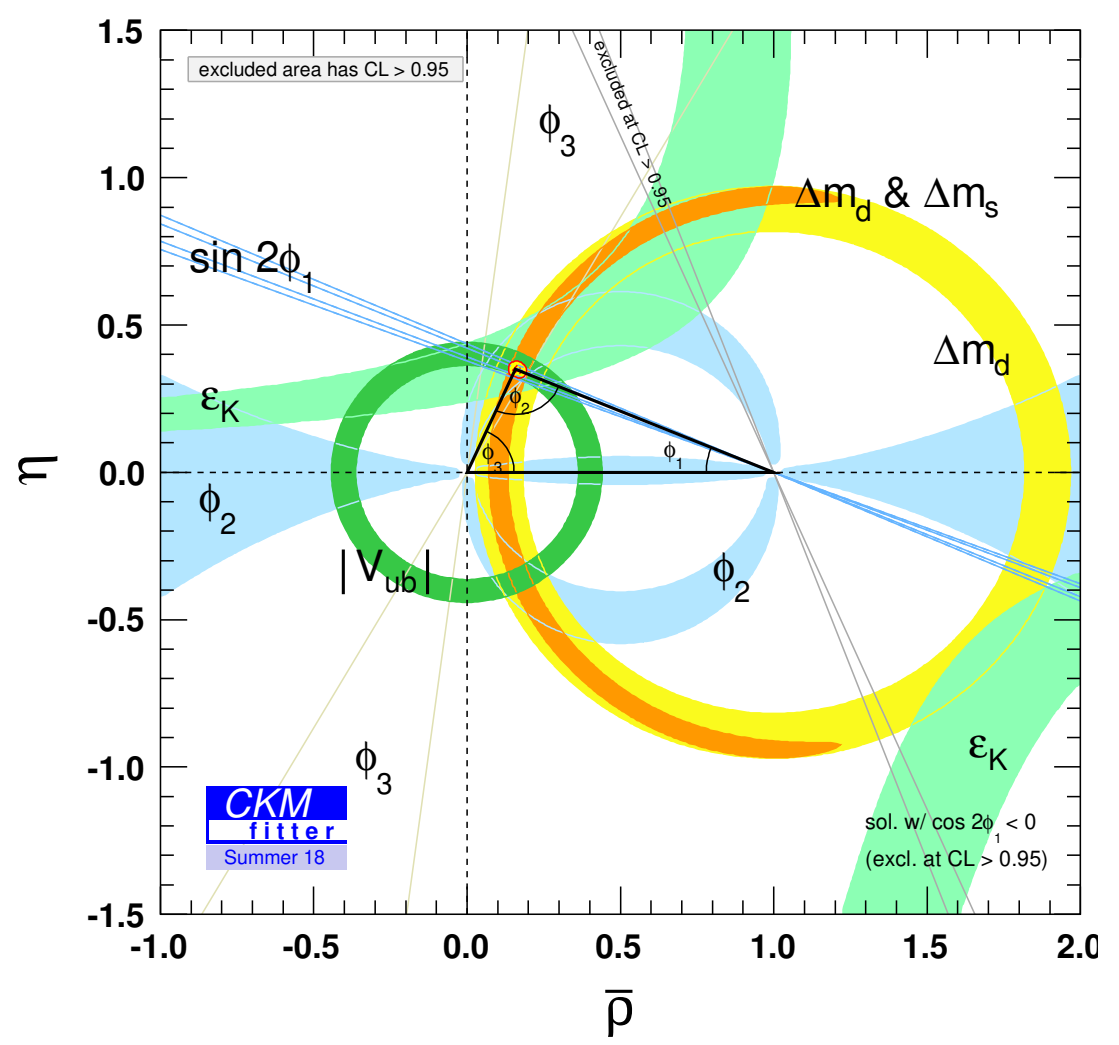

Figure 2: Constraints in the Unitarity Triangle.

through $b \rightarrow c \bar{c} d$ process, where the contribution of the penguin diagram is expected in addition to the dominant tree contribution. Hence, a deviation of $S$ from $-\sin \left(2 \phi_{1}\right)$, where the minus sign comes from the $C P$ eigenvalue of the final state, may help to understand the penguin contribution in this decay, which could also improve the uncertainty of $\sin \left(2 \phi_{1}\right)$ measured with $b \rightarrow c \bar{c} s$. The latter proceeds through the one-loop $b \rightarrow q \bar{q} s$ process, and a deviation of $S$ from $-\sin \left(2 \phi_{1}\right)$ can be a hint of NP.

In both modes, the central values from BaBar [[]] were relatively far from $-\sin \left(2 \phi_{1}\right)$ from $b \rightarrow c \bar{c} s$, though the errors were large. Belle measurement of the $C P$ violation parameters in $B^{0} \rightarrow$ $J / \psi \pi^{0}$ with the full data set of $711 \mathrm{fb}^{-1}$ taken at $\Upsilon(4 S)$ resonance gives $S=-0.59 \pm 0.19 \pm 0.03$ and $A(=-C)=0.15 \pm 0.14_{-0.03}^{+0.04}$ [B]], while, for $B \rightarrow \pi^{0} \pi^{0} K_{S}^{0}$, Belle obtains $S=-0.92_{-0.31}^{+0.27}{ }_{-0.11}^{+0.10}$ and $A=0.28 \pm 0.21 \pm 0.04$ [䧃]. The results are consistent with $\sin \left(2 \phi_{1}\right)$ from $b \rightarrow c \bar{c} s$, though the errors are still large. Search for NP with $C P$ violation in the $b \rightarrow q \bar{q} s$ process are one of the main studies at Belle II experiment, and new results will be expected once Belle II accumulate significant amount of data.

\section{Measurement of $\phi_{s}$}

From the relation of the unitarity of the CKM matrix $V_{u s} V_{u b}^{*}+V_{c s} V_{c b}^{*}+V_{t s} V_{t b}^{*}=0$, one can draw an another squashed triangle in the complex plane. The angle between $V_{c s} V_{c b}^{*}$ and $V_{t s} V_{t b}^{*}$ is called $\beta_{s}$, which can be measured using the mixing-induced $C P$ violation in $B_{s}^{0}$ decays like $B_{s}^{0} \rightarrow J / \psi \phi$. The 
SM prediction gives $\phi_{s} \equiv-2 \beta_{s}=-36.9_{-0.7}^{+1.0} \mathrm{mrad}$ according to the CKMfitter group [ [ $]$ ]. Because of the fast $B_{s}^{0}$ oscillation, an excellent time resolution below $100 \mathrm{fs}$ is necessary to measure $\phi_{s}$, and hence this can be studied only at the hadron colliders where the boost factor is large.

Recent measurement on $B_{s}^{0} \rightarrow J / \psi \phi$ from ATLAS is based on LHC Run 2 data taken at $13 \mathrm{TeV} p p$ collision corresponding to $80.5 \mathrm{fb}^{-1}[$ [G]. In this analysis, the flavor tagging is performed using weighted sum of the charge in a cone around a lepton or in a jet, and is calibrated using the $B^{+} \rightarrow J / \psi K^{+}$control sample. They perform a fit with nine physical parameters including $\phi_{s}$, decay width $\Gamma_{s}$ and its difference $\Delta \Gamma_{s}$, leading to $\phi_{s}=-0.068 \pm 0.038 \pm 0.018 \mathrm{rad}$, where the systematic error mainly comes from the flavor tagging. Combining with the previous result with $19.2 \mathrm{fb}^{-1}$ at 7 and $8 \mathrm{TeV}[$ [ $]$, ATLAS obtains

$$
\begin{aligned}
\phi_{s} & =-0.076 \pm 0.034 \pm 0.019 \mathrm{rad} \\
\Delta \Gamma_{s} & =0.068 \pm 0.004 \pm 0.003 \mathrm{ps}^{-1} .
\end{aligned}
$$

LHCb measures $\phi_{s}$ with $B_{s}^{0} \rightarrow J / \psi K^{+} K^{-}\left[[]\right.$and $B_{s}^{0} \rightarrow J / \psi \pi^{+} \pi^{-}$[Q] using $1.9 \mathrm{fb}^{-1}$ data from LHC Run 2. They obtain $\phi_{s}=-94 \pm 41 \pm 7 \mathrm{mrad}$ from $B_{s}^{0} \rightarrow J / \psi K^{+} K^{-}$and $\phi_{s}=-57 \pm$ $60 \pm 11 \mathrm{mrad}$ from $B_{s}^{0} \rightarrow J / \psi \pi^{+} \pi^{-}$, leading $\phi_{s}=-41 \pm 25 \mathrm{mrad}$ when combined.

Figure B 3 shows the status of measurements of $\phi_{s}$ and $\Delta \Gamma_{s}$ with $b \rightarrow c \bar{c} s$ processes. The experimental average $\phi_{s}=-55 \pm 21 \mathrm{mrad}$ is consistent with the SM prediction $-36.9_{-0.7}^{+1.0} \mathrm{mrad}$, though the current experimental error is still one order larger. The measurements are limited by statistic, and further improvement is expected when more data is available. In fact, the precision at LHCb at High-luminosity LHC with $300 \mathrm{fb}^{-1}$ is expected to be around a few mrad [एत]].

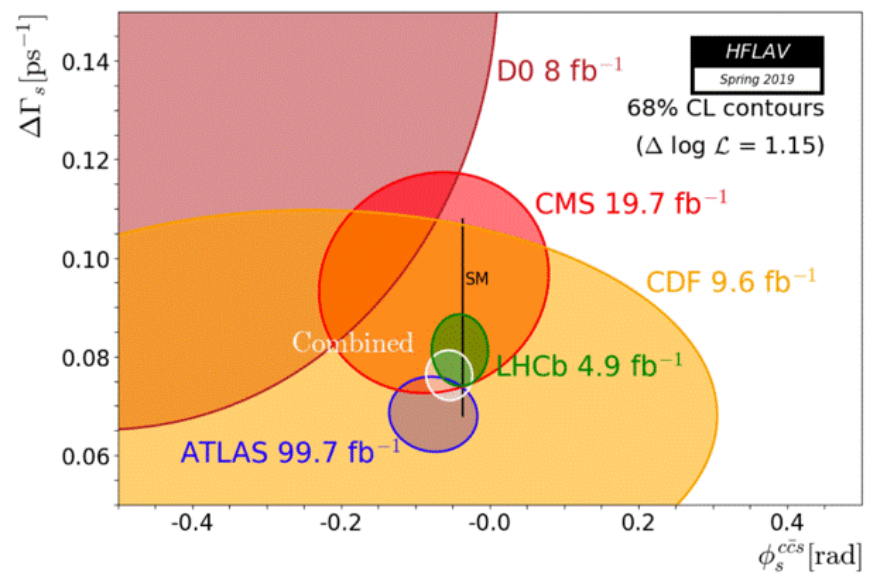

Figure 3: Measurements of $\phi_{s}$ and $\Delta \Gamma_{s}$ with $b \rightarrow c \bar{c} s$ processes.

Similarly to $\phi_{1}$, measurements of $\phi_{s}$ using loop diagram can be a test of NP because of the possible contribution of heavy NP particles in the loop. With $3.0 \mathrm{fb}^{-1}$ data at 7 and $8 \mathrm{TeV}, \mathrm{LHCb}$ has performed a measurement of $\phi_{s}$ in the $b \rightarrow d \bar{d}$ s process using $B_{s}^{0} \rightarrow\left(K^{+} \pi^{-}\right)\left(K^{-} \pi^{+}\right)$decays, and obtains $\phi_{s}^{d \bar{s}}=-0.10 \pm 0.13 \pm 0.14 \mathrm{rad}[\mathbb{W}]$. LHCb has also measured $\phi_{s}$ in the $b \rightarrow s \bar{s} s$ process with $B_{s}^{0} \rightarrow \phi \phi$ to be $\phi_{s}^{s \bar{s} s}=73 \pm 15 \pm 27 \mathrm{mrad}$ [ए]]. Both are consistent with $\phi_{s}$ measurements from $b \rightarrow c \bar{c} s$ and the SM prediction. 


\section{Measurement of $\phi_{3}(=\gamma)$}

The angle $\phi_{3}(=\gamma)$ can be measured using $B \rightarrow D K^{(*)}$ and $B \rightarrow \bar{D} K^{(*)}$. The former proceeds through the color favored tree process with CKM elements $V_{c b} V_{u s}^{*}$, while the latter proceeds through the color suppressed tree process with $V_{u b} V_{c s}^{*}$. When one consider the final states to which both $D$ and $\bar{D}$ can decay, one can extract the weak phase $\phi_{3}$ and $\delta_{B}$ (the strong phase difference of the two amplitudes) from the interference of these two processes. Because the decays are dominated only by tree contributions, the measurement of $\phi_{3}$ is theoretically very clean. There are many $D$ decay modes that can be used to extract $\phi_{3}$, but the amplitude ratio $r_{B}=|A(B \rightarrow \bar{D} K)| /|A(B \rightarrow D K)|$ and the strong phase $\delta_{B}$ are mode-dependent, and the sensitivity to $\phi_{3}$ depends on the modes as well as the central value of $\phi_{3}$.

There are several methods with different $D$ decays to use for the extraction of $\phi_{3}$. The method with multi-body final states common to $D$ and $\bar{D}$ is called GGSZ method [113], which uses the Dalitz plot dependence of the interference. Belle has recently reported a new measurement using $B^{-} \rightarrow D^{0} K^{-}$with $D^{0} \rightarrow K_{S}^{0} \pi^{+} \pi^{-} \pi^{0}$ using the $711 \mathrm{fb}^{-1}$ data set [14]].

The measurement is based on a model-independent formalism via binned Dalitz plot analysis. For the $B^{ \pm}$decay, the partial decay width in $i$ 'th bin can be written as

$$
\begin{aligned}
& \Gamma_{i}^{-}=K_{i}+r_{B}^{2} \bar{K}_{i}+2 \sqrt{K_{i} \bar{K}_{i}}\left(c_{i} x_{-}+s_{i} y_{-}\right) \\
& \Gamma_{i}^{+}=\bar{K}_{i}+r_{B}^{2} K_{i}+2 \sqrt{K_{i} \bar{K}_{i}}\left(c_{i} x_{+}-s_{i} y_{+}\right)
\end{aligned}
$$

where $x_{ \pm}=r_{B} \cos \left(\delta_{B} \pm \phi_{3}\right), y_{ \pm}=r_{B} \sin \left(\delta_{B} \pm \phi_{3}\right)$, and $K_{i}$ and $\bar{K}_{i}$ are the fraction of flavour-tagged $D^{0}$ and $\bar{D}^{0}$ events. $c_{i}$ and $s_{i}$ are the cosine and sine of the strong phase difference between $D^{0}$ and $\bar{D}^{0}$ events, and data from CLEO-c are used as an input for them. $D^{0} \rightarrow K_{S}^{0} \pi^{+} \pi^{-}$would be the primary mode for such studies, but $D^{0} \rightarrow K_{S}^{0} \pi^{+} \pi^{-} \pi^{0}$ is also interesting because it has twice larger branching fraction.

The total number of $B^{-} \rightarrow D^{0} K^{-}$events is $815 \pm 51$. The phase space is divided into 9 bins that are selected so that different internal resonances are contained in different bins. From the fit to Eq. 1.0 and 4.2, the parameters $x_{ \pm}$and $y_{ \pm}$can be extracted. The contour of these parameters is shown in Fig. 国(a). This can be translated into the constraint of $\phi_{3}$ as shown in Fig. 因(b). This is the first measurement of $\phi_{3}$ with this mode, and this measurement improves $\phi_{3}$ measurement by Belle experiment from $\left(78_{-15}^{+14}\right)^{\circ}$ to $\left(74_{-14}^{+13}\right)^{\circ}$. The sensitivity of this analysis at $50 \mathrm{ab}^{-1}$ is $4.4^{\circ}$ [U15], so this mode is promising at Belle II.

LHCb has performed an updated measurement of $\gamma\left(=\phi_{3}\right)$ using $B^{0} \rightarrow D K^{* 0}$ with $D \rightarrow K^{+} \pi^{-}$, $K^{+} K^{-}, \pi^{+} \pi^{-}, K^{+} \pi^{-} \pi^{+} \pi^{-}, \pi^{+} \pi^{-} \pi^{+} \pi^{-}$with $4.8 \mathrm{fb}^{-1}$ from LHC Run 1 and 2 [ए]6]. In terms of the $\gamma$ extraction, the $D$ decay modes $K^{+} \pi^{-}$and $\pi^{+} \pi^{-} \pi^{*} \pi^{-}$work as the GLW method [ㅍ] ], in which the $C P$ eigenstate of $D$ meson is used, while the remaining modes work as the ADS method [ए8]], in which the $C P$ violation is enhanced with doubly Cabibbo suppressed decays.

Figure $\square$ shows the contour plots $\delta_{B}^{D K^{* 0}}$ versus $\gamma$ from this measurement. Currently, the experimental average of $\gamma$ is $\left(71.1_{-5.3}^{+4.6}\right)^{\circ}$ [四], while the average from LHCb measurements is $\left(74.0_{-5.8}^{+5.0}\right)^{\circ}[\mathbb{\square}]$, so the $\gamma$ measurement is dominated by LHCb. This new result is expected to further improve it slightly. 
(a)

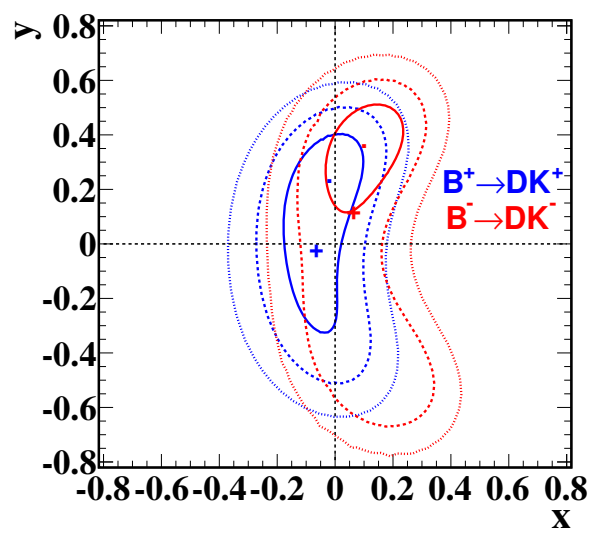

(b)

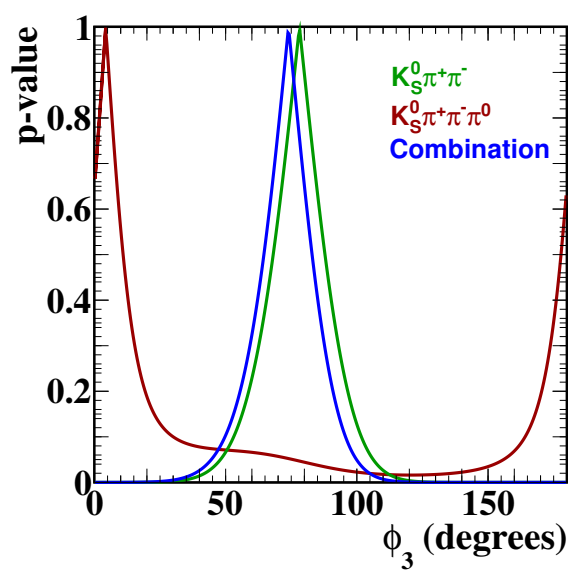

Figure 4: (a) One (solid line), two (dashed line) and three (dotted line) standard deviation likelihood contours for the $\left(x_{ \pm}, y_{ \pm}\right)$parameters for $B^{ \pm} \rightarrow D^{0} K^{ \pm}$decays. The cross marks the expected value from the world average values $\phi_{3}, r_{B}^{D K}, \delta_{B}^{D K}$. (b) Statistical confidence interval for $\phi_{3}$ from Belle with and without this result.

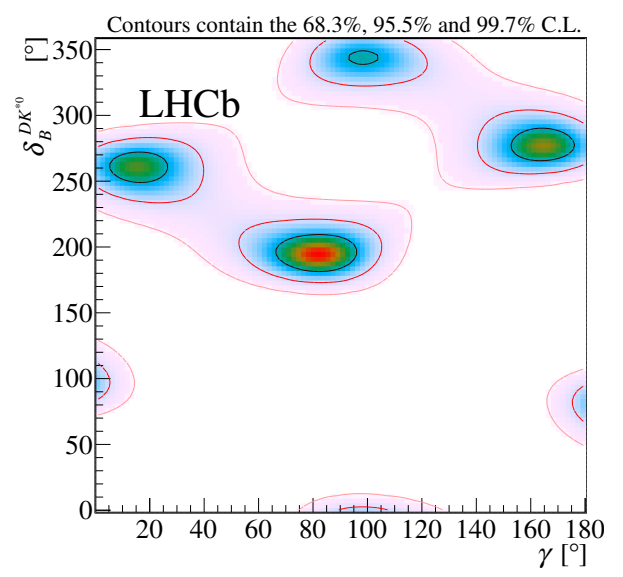

Figure 5: Contour plots over $\delta_{B}^{D K^{* 0}}$ versus $\gamma$ from the LHCb measurement.

\section{Measurement of $\left|V_{c b}\right|$}

The sides of the unitarity triangle $\left|V_{c b}\right|$ and $\left|V_{u b}\right|$ can be measured using semileptonic decays of $B$ mesons through $b \rightarrow c \ell v$ and $b \rightarrow u \ell v(\ell=e, \mu)$ respectively. There are two approaches, inclusive and exclusive, to extract $\left|V_{c b}\right|$ or $\left|V_{u b}\right|$ from these decays. The inclusive approach does not specify the hadron states and measure the parton level decay rate. The exclusive approach specify the final states hadrons, so this approach is experimentally cleaner. Theoretically in the latter case, the decay rate is parametrized in terms of the momentum transfer $q^{2}$ and the form factor is estimated with lattice QCD at high $q^{2}$ and light cone sum rule at low $q^{2}$. There has been a long standing tension between the two approaches as seen in the world average of $\left|V_{c b}\right|$ and $\left|V_{u b}\right|$ shown in Fig.6 [四; in the inclusive approach, $\left|V_{c b}\right|=(42.19 \pm 0.78) \times 10^{-3}$ and $\left|V_{u b}\right|=(4.32 \pm 0.17) \times 10^{-3}$, while in the exclusive approach, $\left|V_{c b}\right|=(39.25 \pm 0.56) \times 10^{-3}$ and $\left|V_{u b}\right|=(3.49 \pm 0.13) \times 10^{-3}$. In both 
$\left|V_{c b}\right|$ and $\left|V_{u b}\right|$, the exclusive approches give around $3 \sigma$ lower values.

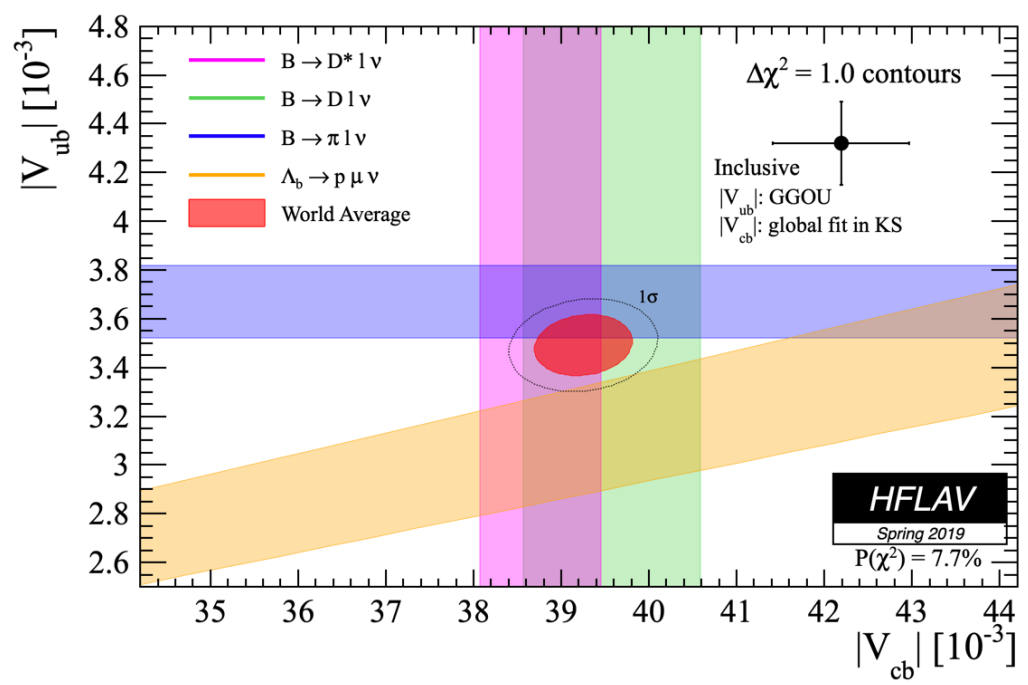

Figure 6: Combined $\left|V_{u b}\right|$ and $\left|V_{c b}\right|$ from exclusive measurements. The average of the inclusive measurements is shown by a data point with error bars.

There exist two major form factor parametrizations: CLN [20] and BGL [2]]. CLN has been mainly used in all the previous measurements, but recently BGL is getting more favored because of the model independent approach. There were some implications that the parametrization might be related to the tension, which suggested the reanalysis of the previous measurements using BGL parametrizaion [22].

Belle has a new result of the untagged analysis of $B \rightarrow D^{*} \ell v$ with $711 \mathrm{fb}^{-1}$ data set [23]]. In this analysis, simultaneous fit to 3 angular variables $\cos \theta_{\ell}, \cos \theta_{V}, \chi$ and hadronic recoil $w$ is performed to extract form factors and $\left|V_{c b}\right|$. The two form factor parametrization CLN and BGL are used for comparison. The obtained results are $\left|V_{c b}\right|=(38.4 \pm 0.2 \pm 0.6 \pm 0.6) \times 10^{-3}$ for CLN parametrization and $\left|V_{c b}\right|=(38.3 \pm 0.3 \pm 0.7 \pm 0.6) \times 10^{-3}$ for BGL parametrization, where the third error is an error from lattice QCD uncertainties. These two values are consistent with each other as well as other exclusive measurements, and are not consistent with inclusive measurements.

In this analysis, Belle has also obtained the branching ratio of electron and muon modes for the lepton flavor universality (LFU) test: $\mathscr{B}\left(B^{0} \rightarrow D^{*-} e^{+} v\right) / \mathscr{B}\left(B^{0} \rightarrow D^{*-} \mu^{+} v\right)=1.01 \pm 0.01 \pm 0.03$. This is the most stringent LFU test in $B$ decays and the result is consistent with unity.

BaBar has performed full 4-dimensional analysis of $B \rightarrow D^{*} \ell v$ using $426 \mathrm{fb}^{-1}$ data set [24]. Hadronic reconstruction with 2968 modes is done for the other side $B$ meson in this analysis. The obtained $\left|V_{c b}\right|$ values are $\left|V_{c b}\right|=(38.40 \pm 0.84) \times 10^{-3}$ for CLN parametrization and $\left|V_{c b}\right|=$ $(38.36 \pm 0.90) \times 10^{-3}$ for BGL parametrization.

In both $\mathrm{BaBar}$ and Belle results, the tension between inclusive and exclusive approaches still persists, after examining both BGL and CLN parametrizations.

\section{6. $C P$ Violation in Three Body Hadronic $B$ Decays}

Search and study of $C P$ violation has been performed in various charmless $B$ decays. $\mathrm{LHCb}$ 
measured the $C P$ asymmetry of $B^{+} \rightarrow \pi^{+} K^{+} K^{-}$to be $\mathscr{A}_{C P}=-0.123 \pm 0.017 \pm 0.012 \pm 0.007$ [ [25], where the last error is due to the $C P$ asymmetry of the reference mode, and found that the asymmetry is localized in the phase space. Belle confirmed it and measured the $K^{+} K^{-}$invariant mass dependence of $\mathscr{A}_{C P}$ [26] .

In order to understand further the source of the $C P$ asymmetry in three body $B$ decays, $\mathrm{LHCb}$ has performed an amplitude analysis of $B^{+} \rightarrow \pi^{+} K^{+} K^{-}$using $3.0 \mathrm{fb}^{-1}$ data taken at LHC Run 1 [27]. They observes $2502 \pm 102$ events for $B^{+} \rightarrow \pi^{+} K^{+} K^{-}$and $1566 \pm 84$ events for $B^{-} \rightarrow$ $\pi^{-} K^{+} K^{-}$. A Dalitz plot fit is performed assuming contributions from five resonance amplitudes, non-resonance amplitude and $\pi \pi \leftrightarrow K K S$-wave rescattering amplitude. The contributions and obtained fractions and $\mathscr{A}_{C P}$ are summarized in Table $\mathbb{W}$. It is found that large $C P$ asymmetry comes from the rescattering component, which can explain the previous results.

Table 1: Result of amplitude analysis on $B^{+} \rightarrow \pi^{+} K^{+} K^{-}$by LHCb. This table is extracted from Table 1 of Ref. [27]

\begin{tabular}{crc}
\hline \hline Contribution & Fit Fraction $(\%)$ & \multicolumn{1}{c}{$A_{C P}(\%)$} \\
\hline$K^{*}(892)^{0}$ & $7.5 \pm 0.6 \pm 0.5$ & $+12.3 \pm 8.7 \pm 4.5$ \\
$K_{0}^{*}(1430)^{0}$ & $4.5 \pm 0.7 \pm 1.2$ & $+10.4 \pm 14.9 \pm 8.8$ \\
Single pole & $32.3 \pm 1.5 \pm 4.1$ & $-10.7 \pm 5.3 \pm 3.5$ \\
$\rho(1450)^{0}$ & $30.7 \pm 1.2 \pm 0.9$ & $-10.9 \pm 4.4 \pm 2.4$ \\
$f_{2}(1270)$ & $7.5 \pm 0.8 \pm 0.7$ & $+26.7 \pm 10.2 \pm 4.8$ \\
Rescattering & $16.4 \pm 0.8 \pm 1.0$ & $-66.4 \pm 3.8 \pm 1.9$ \\
$\phi(1020)$ & $0.3 \pm 0.1 \pm 0.1$ & $+9.8 \pm 43.6 \pm 26.6$ \\
\hline \hline
\end{tabular}

LHCb also performs an amplitude analysis for $B^{+} \rightarrow \pi^{+} \pi^{-} \pi^{+}$with $3.0 \mathrm{fb}^{-1}$ data taken at LHC Run 1 [28]. In this analysis, five resonant components $\rho(770)^{0}, \omega(782), f_{2}(1270), \rho(1450)^{0}$, $\rho_{3}(1690)^{0}$ and $S$-wave comonent are considered. In order to describe the $S$-wave component, three different approaches (isobar model, K-matrix formalism, quasi-model-independent approach) are used. From the fit to the Dalitz plot, large $C P$ violation is found in $f_{2}(1270)$ and $S$-wave component. Additionaly, from the analysis on the helicity angle distribution, $C P$ violation is also found in $\rho(770)^{0}$-scalar inteference.

These new amplitude analyses may give new picture on the $C P$ violation in hadronic $B$ decays. $\mathrm{LHCb}$ can do more studies on this topic with Run2 data.

Belle may not be competetive to LHCb with these modes but has advantage for modes with neutral particles in the final states. Belle has performed a study on $B^{+} \rightarrow K_{S}^{0} K_{S}^{0} K^{+}$[ㅁ] and has obtained $\mathscr{B}\left(B^{+} \rightarrow K_{S}^{0} K_{S}^{0} K^{+}\right)=(6.5 \pm 2.6 \pm 0.4) \times 10^{-7}$ and $\mathscr{A}_{C P}\left(B^{+} \rightarrow K_{S}^{0} K_{S}^{0} K^{+}\right)=(1.6 \pm 3.9 \pm$ $0.9) \times 10^{-2}$. This is not an amplitude analysis, but the branching fraction and $C P$ asymmetry in bins of $M_{K_{S}^{0} K_{S}^{0}}$ are examined. The amplitude analysis will be possible at Belle II, once an order larger data than Belle is accumulated.

\section{Summary}

Recent measurements related to CKM matrix elements and $C P$ violation in 3 body $B$ decay have been summarized. There has been a significant improvement on the precision of $\beta_{s}$, though 
the experimental error is still large compared to the theoretical uncertainty. The discrepancy of $\left|V_{c b}\right|$ in exclusive and inclusive measurement is still unchanged, and this should be understand to pursue $\mathrm{NP}$ contribution from the unitarity triangle. In near future, more results from $\mathrm{LHCb}$ are expected and Belle II will join the game for more precise measurements.

\section{References}

[1] The Heavy Flavor Averaging Group (HFLAV), https://hflav . web.cern.ch/.

[2] B. Aubert et al. (BaBar Collaboration), Phys. Rev. Lett. 101, 021801 (2008); B. Aubert et al. (BaBar Collaboration), Phys. Rev. D76, 071101 (2007).

[3] B. Pal et al. (Belle Collaboration), Phys. Rev. D98, 112008 (2018).

[4] Y. Yusa et al. (Belle Collaboration), Phys. Rev. D99, 011102 (2019).

[5] CKMfitter Group, http://ckmfitter.in2p3.fr/.

[6] ATLAS Collaboration, ATLAS-CONF-2019-009.

[7] A. Georges et al. (ATLAS Collaboration), JHEP 08, 137 (2016).

[8] R. Aaij et al. (LHCb Collaboration), Eur. Phys. J. C79, 706 (2019).

[9] R. Aaij et al. (LHCb Collaboration), Phys. Lett. B797, 134789 (2019).

[10] R. Aaij et al. (LHCb Collaboration), arXiv:1808.08865.

[11] R. Aaij et al. (LHCb Collaboration), JHEP 03, 140 (2018).

[12] R. Aaij et al. (LHCb Collaboration), arXiv:1907.10003.

[13] A. Giri, Y. Grossman, A. Soffer and J. Zupan, Phys. Rev. D68, 054018, (2003); A. Bondar, talk at the Belle analysis workshop, Novosibirsk, September 2002.

[14] P. Resmi et al. (Belle Collaboration), arXiv:1908.09499.

[15] E. Kou et al., arXiv:1808.10567.

[16] R. Aaij et al. (LHCb Collaboration), JHEP 09, 041 (2019).

[17] M. Gronau and D. London, Phys. Lett. B253, 483 (1991); M. Gronau and W. Daniel, Phys. Lett. B265, 172 (1991).

[18] D. Atwood, I. Dunietz and A. Soni, Phys. Rev. Lett. 78, 3257 (1997); D. Atwood, I. Dunietz and A. Soni, Phys. Rev. D63, 036005 (2001).

[19] M. Kenzie and M. Whiteboard (LHCb Collaboration), LHCb-CONF-2018-002.

[20] I. Caprini, L. Lellouch and M. Neubert, Nucl. Phys. B530, 153 (1998).

[21] C. G. Boyd, B. Grinstein and R. F. Lebed, Phys. Rev. Lett. 74, 4603 (1995).

[22] D. Bigi, P. Gambino and S. Schacht, Phys. Lett. B769, 441 (2017); B. Grinstein and A. Kobach, Phys. Lett. B771, 359 (2017).

[23] A. Abdesselam et al. (Belle Collaboration), arXiv:1809.03290.

[24] J. P. Lees et al. (BaBar Collaboration), Phys. Rev. Lett. 123, 091801 (2019).

[25] R. Aaij et al. (LHCb Collaboration), Phys. Rev. D90, 112004 (2014). 
[26] C. Hsu et al. (Belle Collaboration), Phys. Rev. D96, 031101 (2017).

[27] R. Aaij et al. (LHCb Collaboration), arXiv:1905.09244.

[28] R. Aaij et al. (LHCb Collaboration), arXiv:1909.05211, R. Aaij et al. (LHCb Collaboration), arXiv:1909.05212.

[29] A. Kaliyar et al. (Belle Collaboration), Phys. Rev. D99, 031102 (2019). 